\title{
Invited review: Quantitative trait nucleotide determination in the era of genomic selection
}

\author{
J. I. Weller ${ }^{1}$ and M. Ron \\ Institute of Animal Sciences, A.R.O., The Volcani Center, Bet Dagan 50250, Israel
}

\section{ABSTRACT}

Genome-wide association studies based on tens of thousands of single nucleotide polymorphisms have been completed for several dairy cattle populations. Methods have been proposed to directly incorporate genome scan data into breeding programs, chiefly by selection of young sires based on their genotypes for the genetic markers and pedigree without progeny test. Thus, the rate of genetic gain is increased by reduction of the mean generation interval. The methods developed so far for application of genomic selection do not require identification of the actual quantitative trait nucleotides (QTN) responsible for the observed variation of quantitative trait loci (QTL). To date, 2 QTN affecting milk production traits have been detected in dairy cattle: DGAT1 and ABCG2. This review will attempt to address the following questions based on the current state of bovine genomics and statistics. What are the pros and cons for QTN determination? How can data obtained from high-density, genome-wide scans be used most efficiently for QTN determination? Can the genome scan results already available and next-generation sequencing data be used to determine QTN? Should QTN be treated differently than markers at linkage disequilibrium with QTL in genetic evaluation programs? Data obtained by genome-wide association studies can be used to deduce QTL genotypes of sires via application of the a posteriori granddaughter design for concordance testing of putative QTN. This, together with next-generation sequencing technology, will dramatically reduce costs for QTN determination. By complete genome sequencing of 21 sires with many artificial insemination sons, it should be possible to determine concordance for all potential QTN, thus establishing the field of QTNomics.

Key words: dairy cattle, genomic selection, markerassisted selection, quantitative trait nucleotide

Received September 7, 2010.

Accepted November 9, 2010.

${ }^{1}$ Corresponding author: weller@agri.huji.ac.il

\section{INTRODUCTION}

Beginning in 1995 (Georges et al., 1995), daughter and granddaughter designs have been applied to nearly all major dairy cattle populations to detect the QTL affecting economic traits (reviewed by Weller, 2007). However, confidence intervals for QTL detected by this methodology extend over tens of map units. Until 2000, identification of the actual quantitative trait nucleotides (QTN) was possible only for microbes, plants, and model organisms. This is no longer the case. At least 4 QTN have been identified and verified by multiple studies in farm animals (Ron and Weller 2007). Of these, 2 are in dairy cattle, DGAT1 and ABCG2 (Grisart et al., 2002; Winter et al., 2002; Cohen Zinder et al., 2005). The most significant effects for both genes are on fat and protein concentrations, which have the highest heritabilities of all traits routinely analyzed in dairy cattle.

Ron and Weller (2007) presented a schematic strategy for QTN determination and validation in livestock. In view of the revolution of genomic selection in dairy cattle, a timely question arises - although QTN can be identified, is it worth the effort? In this review the current state of genomic selection and QTN determination in dairy cattle will be presented. We will consider how data obtained from genome-wide association studies (GWAS) can also be used for QTN determination. Within this context we will try to evaluate the advantages and disadvantages of QTN determination.

\section{WITHIN-FAMILY LINKAGE VERSUS POPULATION- WIDE LINKAGE DISEQUILIBRIUM}

Unlike genetic linkage within families that extends over tens of centimorgans, population-wide linkage disequilibrium (LD) extends in dairy cattle over less than 1 cM (McKay et al., 2007; Sargolzaei et al., 2008). Meuwissen and Goddard (2000) proposed that confidence intervals for QTL location could be dramatically reduced by application of population-wide LD mapping. Application of LD therefore requires much denser genetic maps than are required for detection of QTL via linkage, but does not require a specific family structure 
(Grapes et al., 2004). Since the first reports of GWAS based on dense arrays of SNP in 2006 (Goddard et al., 2006), all studies agree that based on the criterion of the false discovery rate (Weller et al., 1998), many more statistically significant SNP effects have been found compared with linkage-based genome scans (Loberg and Dürr, 2009). The apparent reasons are as follows:

1. Genome coverage with tens and hundreds of thousands of SNP is more complete.

2. Unlike granddaughter or daughter designs (Weller et al., 1990), which can only use bulls from large half-sib families, all sires with evaluation can be included in LD analyses. Thus, sample sizes are greater.

3. Linkage disequilibrium effects can be tested by a simple linear model of number of "+" alleles on the bulls' genetic evaluations or daughter yield deviations (DYD; e.g., Szyda et al., 2009). This test has higher statistical power compared with linkage studies.

In January 2008, Illumina Inc. (San Diego, CA) announced release of the Infinium BovineSNP50 BeadChip, which includes 54,001 SNP approximately evenly spaced across the entire bovine genome. The results of VanRaden et al. (2009) for the US Holstein population confirm that, at least with respect to the QTN that have been detected, results of GWAS do correspond to the results of daughter and granddaughter designs. The largest effect found for fat concentration was located on BTA14 flanking the DGAT1 gene, with lesser effects on milk and fat yields. Similarly, the largest effect found for protein concentration was on BTA6 flanking the ABCG2 gene. Cole et al. (2009) confirmed the QTN for both genes on BTA6 and BTA14 in an analysis of 5,285 US Holstein bulls.

Results of 912 Israeli Holstein bulls show that of the 8 effects with the lowest probabilities for fat concentration, 7 were located on BTA14 in the vicinity of DGAT1. The SNP with the lowest probability was located at position 443,937 bp on BTA14, 1,149 bp from the QTN (Grisart et al., 2002). Similarly, the SNP with the most significant effects on protein concentration formed a bracket from $37,024,132$ to $37,454,409$ bp on BTA6. The ABCG2 QTN is located within this bracket, 36,301 bp from its higher end (Cohen-Zinder et al., 2005). In both cases, GWAS identified segregating QTL within distances $<40 \mathrm{kbp}$ from the QTN. Furthermore, no additional effects accounting for larger fractions of the genetic variances than the identified QTN were found for any of the traits analyzed.

\section{APPLICATION OF LD GENOME SCANS TO BREEDING PROGRAMS}

Application of marker-assisted selection (MAS) based on GWAS requires solutions to new statistical problems. Specifically, how should information from pedigree, phenotypic records, and genotypes be combined to optimally rank candidates for selection? Goddard and Hayes (2007) proposed that genomic selection could be divided into 3 steps:

1. Use the markers to deduce the genotype of each animal at each QTL;

2. Estimate the effects of each QTL genotype on the trait;

3. Sum all the QTL effects for selection candidates to obtain their genomic estimated breeding values (GEBV).

VanRaden (2008) proposed that steps 1 and 2 could be bypassed by analysis of DYD as the dependent variable with all SNP included as random effects. Predictions were computed using linear and Bayesian genomic models. For linear predictions, the traditional additive genetic relationship matrix was replaced by a genomic relationship matrix, which is equivalent to assigning equal genetic variance to all markers. For Bayesian predictions, markers with smaller effects were regressed further toward zero. Final GEBV are derived by combining the following 3 terms by selection index:

1. Direct genomic prediction,

2. Parent averages computed from the set of genotyped ancestors using traditional relationships,

3. Published parent averages or pedigree indexes, constructed as 0.5 (sire GEBV) + 0.25 (maternal grandsire GEBV) + 0.25(birth year mean GEBV).

Combined predictions were more accurate than official parent averages for all 27 traits analyzed (VanRaden et al. 2009). Reliabilities were 0.02 to 0.38 higher with nonlinear genomic predictions included compared with parent averages alone. Genomic EBV have been published in the US since April 2008. Nearly all of the top US bulls are currently young bulls with GEBV but without progeny tests.

Variations of this method have also been applied to other national dairy cattle populations (e.g., Ducrocq and Liu, 2009). Other methodologies have been proposed, but almost all are based on analysis of DYD or genetic evaluations (Loberg and Dürr, 2009). Weak- 
nesses of these methods were discussed by Aguilar et al. (2010) and Misztal et al. (2010). They developed a method for direct incorporation of genomic information into a standard animal model evaluation of the entire national population. Genomic information is incorporated via an augmented relationship matrix. None of these methods assume or require that any of the actual QTN have been identified.

\section{HOW MANY QTL ARE DETECTABLE?}

No assumptions are made in the schemes proposed for derivation of GEBV with respect to the actual architecture of the QTL. Hayes et al. (2006) estimated that the number of detectable QTL affecting milk production is on the order of 150, based on GWAS with 10,000 SNP, whereas Chamberlain et al. (2007) estimated the total number of QTL at 30 by analysis of a daughter design. These studies proposed that most of the additive genetic variance can be explained by QTL that can be detected by GWAS, provided that the number of animals analyzed and the SNP densities are sufficient.

Although the US analysis of 5,285 bulls confirmed the genes on BTA6 and BTA14, it revealed few other large effects. Markers on BTA18 had the largest effects on calving ease, several conformation traits, longevity, and total merit (Cole et al., 2009). Of the 31 traits analyzed for Holsteins, only 14 effects $>0.1$ genetic standard deviations were found (http://aipl. arsusda.gov/Report_Data/Marker_Effects/marker_effects.cfm? Breed = HO). Weigel et al. (2009) compared GEBV computed with all valid SNP markers to GEBV computed with subsets of markers. The coefficients of determination for subsets of the 300, 500, 750, 1,000, $1,250,1,500$, and 2,000 SNP with largest effects were $0.184,0.236,0.279,0.289,0.307,0.313$, and 0.322 , respectively. These results tend to validate quantitative genetic assumptions that the genetic variances of most traits are due to the contribution of a large number of genes each of small effect.

If this is the case, it is reasonable to ask how efficient current SNP chips are in explaining the genetic variation of a given trait. This question was considered for humans with respect to height and several highheritability disease traits (Maher, 2008). Three groups of researchers conducted GWAS of huge populations for height, a high-heritability trait. More than 40 effects were verified by detection in independent analyses, but these altogether accounted for little more than $5 \%$ of the phenotypic variance. Similar results were found for autism and schizophrenia, even though both traits also have very high heritability.

Various explanations have been advanced to explain these disappointing results. First, sample sizes of tens of thousands of individuals are required to obtain statistical power to detect QTL explaining $<0.5 \%$ of the total variance (Visscher, 2008, 2010). For example, with a sample size of 10,000 , statistical power to detect a variant that explains $0.2 \%$ of the variance is only $29 \%$. Therefore, the chance that 2 independent studies of this size both detect this variant is only $0.29^{2}=0.08$. A significant fraction of the genetic variation may be due to rare alleles, which are very difficult to detect (Maher, 2008). Finally, a large fraction of the genetic variation in quantitative traits may be due to polymorphisms in DNA that will not be detected by linkage to SNP; for example, copy number variation (Maher, 2008).

With respect to cattle, the statistical power for QTL determination is higher per individual genotyped because of the availability of high reliability breeding values for thousands of sires, much smaller effective population size, and larger extent of LD in cattle compared with human populations. As a consequence, the heritability of the traits has only a marginal effect on the statistical power to detect QTN. On the other hand, most of the traits of interest in cattle have been under intense selection for several generations. This should result in rapid fixation of the segregating QTL with the largest effects. For example, in the last 2 decades, the main trait under selection was protein production. The largest effect detected for this trait in the US Holstein analysis was only 0.02 genetic standard deviations (http://aipl. arsusda.gov/Report_Data/Marker_Effects/marker_ effects.cfm?Breed = HO).

\section{APPLICATION OF IDENTIFIED QTL IN MAS}

For a QTL to be useful in a MAS program it must fulfill all of the following criteria:

1. Due to the huge multiple comparison problem, confidence that a segregating QTL has in fact been detected is only obtained if the observation is repeated in several independent studies (Lander and Kruglyak, 1995).

2. The effect should be sufficiently large so that the confidence interval for QTL location is small enough so that a haplotype including the QTL can be determined and followed through pedigrees.

3. The net effect of the QTL on the selection index must be significant.

4. Scope for selection is possible only if the frequency of the economically positive allele is relatively low.

It is quite difficult to find a single reported QTL that meets all these criteria. For example, of the 2 QTN that 
have been determined in cattle, DGAT1 fails the third criterion and $A B C G 2$ fails the fourth criterion. The allele of DGAT1 that increases fat production reduces protein; thus, the net effect on most selection indices will be close to zero (Weller et al., 2003). For $A B C G 2$, one allele is clearly economically favorable for almost all selection indices currently in use, but this allele is already at a very high frequency in all dairy cattle populations analyzed (Ron et al., 2006).

Thus, the arguments against extending significant effort toward QTN detection are daunting, and can be summarized as follows:

1. The infinitesimal model appears to be approximately accurate for the traits of interest. That is, genetic variance is apparently due to the joint effect of many genes, all with very small effects.

2. Even if a QTN is detected, it may not be useful in selection, either because its net effect on the selection index is negligible, or because the economically favorable allele is already at high frequency in the population.

3. The methods derived so far for genomic selection appear to work well.

4. Detection of QTN is expensive and time consuming, especially if the effect is due to a more complicated genetic mechanism, such as copy number variation or DNA methylation.

\section{FUTURE PROSPECTS FOR QTN DETERMINATION}

It took $10 \mathrm{yr}$ from the first detection of a segregating QTL on BTA6 until identification of the causative mutation (Cohen-Zinder et al., 2005), whereas for DGAT1 this process took 4 yr (Grisart et al., 2002; Winter et al., 2002). Clearly, it is unlikely that in the future this much time or effort can be justified for QTN identification. Darvasi (2005) wrote: "With the availability and constant production of novel mouse genomic tools, I have no doubt that in the future dissecting a quantitative phenotype into its underlying genes will become a routine task achieved in a timeframe of one year possibly in 80 days." Although this statement refers to experimental animals, specifically mice, the tools available for QTN determination have made the 1-yr goal realistic even for farm animals, and especially for dairy cattle. Meta-analyses of QTL mapping in cattle (Khatkar et al., 2004; Salih and Adelson, 2009), the annotated bovine genome (Btau 4.0), the accumulation of data on gene expression and function in cattle (Keane et al., 2009; Ogorevc et al., 2009), high-density genotyping arrays (Illumina Inc.) and next-generation sequencing technologies (Roche 454; Illumina Genome Analyzer,
ABI-SOLiD, and others) are major developments that will accelerate the process of QTN determination.

As noted by Ron and Weller (2007) the most convincing proof of QTN determination is "concordance." That is, to demonstrate for a group of animals that their genotypes for the putative QTN correspond to their inferred genotypes for the QTL. In dairy cattle, QTL genotypes can be determined for sires with many progeny by application of either a daughter or a granddaughter design. The number of sires for which QTL genotype has been determined must be sufficiently large so that the hypothesis of obtaining concordance by chance within the QTL confidence interval can be rejected with reasonable statistical power. Approximately 15 animals with QTL genotypes determined are sufficient to reject the hypothesis of concordance by chance (Ron and Weller, 2007).

\section{DETERMINATION OF CONCORDANCE BY THE A POSTERIORI GRANDDAUGHTER DESIGN}

Genome-wide association studies can be a major tool in QTN determination. Once genomic regions containing segregating QTL have been determined by GWAS, the QTL genotype of the sires with many sons included in the sample can be determined with high certainty by application of an a posteriori granddaughter design (APGD). That is, for each of the sires, haplotypes can be determined for a chromosomal segment including a SNP with a major effect on an economic trait. The sons can be divided into 2 groups, based on which paternal haplotype was received. Based on the difference between the mean DYD of the 2 groups of sons, it should be possible to determine with relative certainty if their sire was heterozygous or homozygous for the QTL (Israel and Weller, 2004).

Application of the APGD can be illustrated with the following example. Of 912 bulls genotyped in Israel, 24 were sons of sire 829 , who was heterozygous for the causative mutation in $A B C G 2$ on BTA6. Based on 4 heterozygous SNP between positions 37.0 and 37.3 million bp, we were able to determine which paternal haplotype was passed to 22 of the sons. The difference between the means of the genetic evaluations of the 2 haplotype groups was $0.183 \pm 0.040 \%$ protein, which corresponds closely to the effect of $0.2 \%$ protein estimated by Cohen-Zinder et al. (2005). The largest effect associated with any of the individual markers in this chromosomal region was $0.147 \pm 0.024 \%$ protein.

Unlike the traditional granddaughter design, all families analyzed by the APGD will be informative, as illustrated in Figure 1. Concordance requires that a significant contrast should be obtained for all sires 


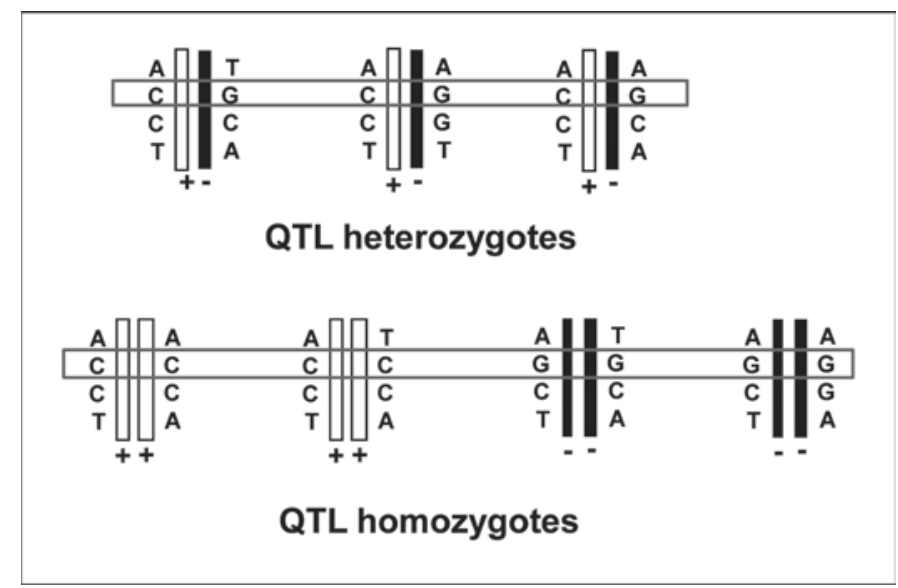

Figure 1. The a posteriori granddaughter design. Three grandsires heterozygous for the QTL and 4 grandsires homozygous for the QTL are illustrated. Chromosomes with the positive QTL allele are white, and chromosomes with the negative QTL allele are black. The QTL alleles are indicated under the homologous chromosomes as either "+" or "_". Genotypes for 4 adjacent SNP are given. Concordance of grandsire SNP genotypes with QTL genotypes is obtained only for the second SNP, and this SNP is enclosed in a rectangle.

heterozygous for the marker, and the same allele should have the positive effect in all families. Similarly, for those sires homozygous for the putative QTN, it will still be possible to distinguish between their haplotypes based on flanking markers, and to determine which haplotype was passed to each of their sons. If a sire is homozygous for the QTL, it will not be possible to determine accurately if this sire is homozygous for the positive or negative allele (Israel and Weller, 2004), but any marker that is heterozygous in these sires can be

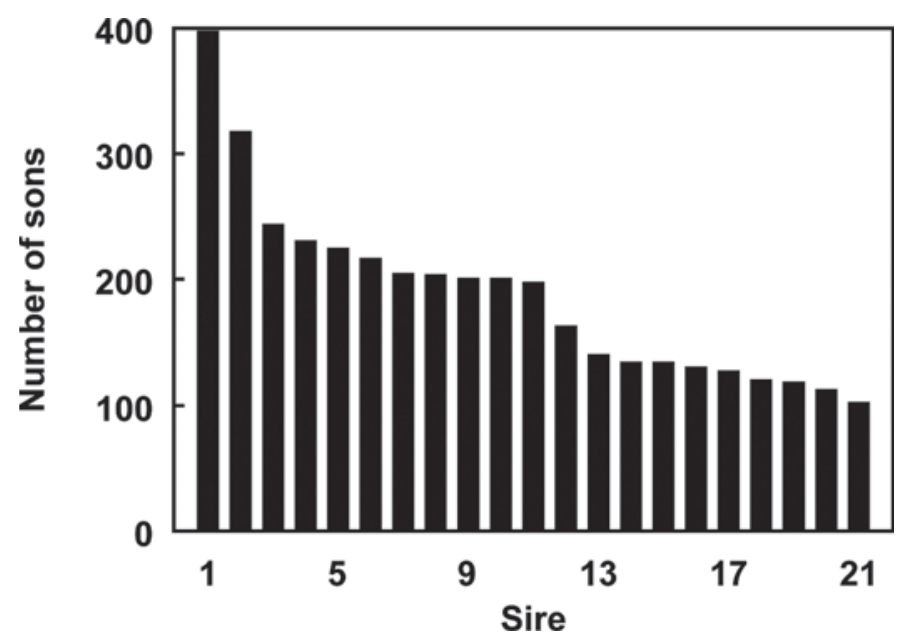

Figure 2. The numbers of US Holstein sons per sire with genotypes for the BovineSNP50 BeadChip (Illumina Inc., San Diego, CA) and genetic evaluations for milk production traits. Data from the May 2010 Interbull pedigree file. excluded as the QTN. Similarly, if a sire is homozygous for a specific marker, and the contrast between the haplotypes is significant for the quantitative trait, then this marker can be excluded as the QTN.

The APGD may be directly applied to the US population based on available data. Of the 60,974 US Holstein bulls with identified sires listed in the May 2010 Interbull pedigree file, 12,626 have been genotyped for the BovineSNP50 BeadChip. Twenty-one of the sires of these bulls have from 100 to 400 sons with genotypes and genetic evaluations (Figure 2). The QTL regions with the most significant effects on traits of economic importance may be analyzed based on the genotypes of the 21 US sires with the greatest number of genotyped sons with genetic evaluations. This sample is clearly sufficient to determine concordance for a putative QTN.

Application of the APGD will have very useful byproducts:

1. One of the weaknesses of single-marker LD analysis of quantitative traits is that when a significant effect is detected, it will generally be biased due to multiple comparisons (Weller et al., 2005) and will also be confounded with additive polygenic effects. That is, bulls with common SNP genotypes will also tend to be related. In the extreme case, a rare SNP allele may be found only in a single extended family, due to a mutation in a single sire. If this individual is also extreme for the trait analyzed, then this SNP will display a significant effect on the trait, even if there is no segregating QTL in the vicinity. By application of the APGD, all effects not linked to the chromosomal segment analyzed should be randomly distributed among the 2 progeny groups.

2. Unless the actual QTN was by chance included in the genome scan, the effects detected by LD analysis will underestimate the actual effect, because association between the SNP and the causative site will be incomplete. However, this will not be the case for the difference between 2 groups of sons that inherit the 2 paternal haplotypes. Because the QTL has been localized by LD to a chromosomal segment of less than $1 \mathrm{cM}$, reduction of the QTL effect in the sons due to recombination will be insignificant.

3. The new analysis, based on contrasts of haplotypes within families, is sufficiently different from the preliminary LD analysis so that it can be considered a nearly independent confirmation of the effect detected.

4. Because a specific chromosomal region is targeted, the multiple comparisons problem is re- 


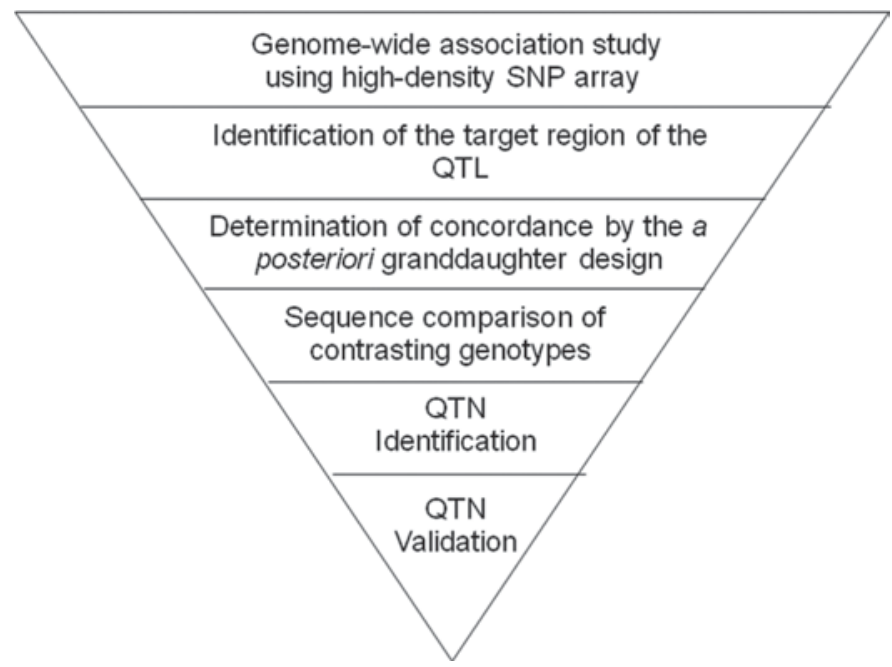

Figure 3. The proposed scheme from genome-wide association study to quantitative trait nucleotide (QTN) identification in cattle.

duced, both with respect to the significance level required and bias (Weller, 2009).

\section{THE PROPOSED SCHEME FROM GWAS TO QTN DETERMINATION IN CATTLE}

Even if 54,001 SNP are included in the genome scan, very few will be QTN for detectable QTL. In 2010, Illumina released the BovineHD genotyping BeadChip with $>777,000$ SNP with an average gap size of 3.43 kbp. Currently, 17,999,182 known SNP exist for humans (http://www.ensembl.org/Homo_sapiens/Info/ StatsTable). Assuming a similar number of SNP in cattle, if there are 50 genes with detectable segregating effects on each quantitative trait and 20 traits are analyzed, only 1 SNP in approximately 15,000 will be a QTN. Thus, the expectation is that only 2 to 3 detectable QTN will be included within a sample of 54,000 SNP and 30 to 45 detectable QTN within a sample of 777,000 SNP. Therefore, after major segregating QTL have been determined by a linear model analysis and verified by the APGD, most QTN should be identified. For the remainder of unidentified QTN, it will still be necessary to genotype additional markers within the confidence interval to find the actual QTN.

With the development of next-generation sequencing technologies, tens of thousands of base pairs can be readily compared between individuals with opposing genotypes for the QTL (Mardis, 2008; Yeager et al., 2009). Thus, the entire critical region of association delimited by margins that show substantial decay of LD can now be sequenced to determine putative QTN. In addition, considering the expected reduction in complete genome resequencing costs (Pérez-Enciso and
Ferretti, 2010; http://genomics.energy.gov/), a complete DNA sequencing is feasible for bulls that display the highest number of contrasting genotypes for the QTL. These data may be combined with imputation techniques to derive almost complete genotypes for bulls analyzed by high-density SNP chips (Druet and de Roos, 2010; Hickey et al., 2010; Weigel et al., 2010; Zhang and Druet, 2010). Candidate QTN will be validated by analysis of the remaining bulls with imputed genotypes for the QTL in concordance tests.

Ron and Weller (2007) proposed that because positional cloning is labor intensive, the "best" candidate genes should be analyzed first, based on knowledge of the positional genes' function and expression patterns. However, it must be noted that results of the candidate gene approach in the analysis of the genetic control of human diseases have been "woefully inadequate" (Altshuler et al., 2008). The criteria for selection of targets for QTN identification are as follows:

1. Identification of a concise genomic region spanning less than 1 million bp with several SNP markers with significant LD to the trait, and defined margins that show substantial decay of LD. Because of the huge number of SNP analyzed, the effect should be significant at the $5 \%$ level under the Bonferroni criterion of significance.

2. Because all analysis models are problematic, significance should be confirmed by application of several different models to the same data.

3. The estimated effect should be sufficiently large to have economic value. A reasonable criterion would be an effect that explains at least $1 \%$ of the genetic variance of the selection index.

4. Priority should be given to regions determined to harbor segregating QTL from meta-analyses (Khatkar et al., 2004; Salih and Adelson, 2009).

5. For selection to be effective, the frequency of the economically favorable allele should be low. Although the SNP analyzed will generally not be the putative QTN, and the allelic frequency of the SNP will not be identical to that of the QTN, it is likely that if LD is very strong, then frequencies of the alleles for the SNP should approximate QTN frequencies.

In Figure 3 the proposed scheme from GWAS to QTN identification in cattle is presented. The main features of this scheme are

1. Application of a genome scan, using a dense matrix of SNP markers.

2. Estimation of the effects of each SNP on the economic traits. 
3. Determination of target regions based on statistical analysis and previous data.

4. Determination of the physical map of genes in the target regions.

5. Determination of haplotypes of sires with many sons.

6. Deduction of QTL genotypes of these sires by the APGD.

7. Application of next-generation sequencing technology to resequence DNA samples of sires with alternate QTL genotypes.

8. Determination of concordance of sires by the APGD for putative QTN.

9. Validation of the QTN effect by functional studies.

\section{IS QTN DETERMINATION WORTH THE EFFORT?}

Ron and Weller (2007) termed the process from QTL to QTN identification "Winning by points rather than knock-out." Although this notion still holds, all features in the scheme to identify QTN except the last one should be now more efficient than the scheme proposed. So far, no short-cuts have been found for this final complex step of validation. Additional laboratory analyses required beyond GWAS will be DNA resequencing of individual sires with alternate genotypes for QTL and validation of the putative QTN by functional studies. Future costs of new technologies will always be considerably lower than the current costs. Complete Genomics (Mountain View, CA) plans to offer complete human genome sequence per individual for US $\$ 5,000$ next year (Hayden, 2009). With similar costs, 21 sires with many AI sons could be sequenced for approximately $\$ 100,000$. This cost is, of course, negligible compared with the huge costs already spent for generating genotypes of tens of thousands of sires. This would make possible concordance determination for all potential QTN detected by GWAS. Thus, despite the arguments against QTN determination given above, several justifications can be given:

1. Once the QTN is determined, this will yield useful information on gene function and QTL architecture. Although the 2 QTN determined in dairy cattle are both missense mutations, this is not the case for the 2 QTN determined in other farm animal species (Ron and Weller, 2007). Thus, it is still not clear if missense mutations are the exception or the rule for QTN in commercial animals. Further efforts toward novel QTN discovery may resolve this dilemma.
2. Understanding the ties between genetic variation and functional characteristics of specific genes may contribute to drug discovery for the benefit of both farm animals and humans.

3. As demonstrated for the case of $A B C G 2$, although SNP in close linkage to a major QTN will generally display highly significant effects, the effect will still be significantly less than the effect obtained with the QTN (Cohen-Zinder et al., 2005; Olsen et al., 2007). Thus, selection on the QTN may be more efficient than selection on a marker in LD (i.e., genomic selection).

4. Linkage disequilibrium relationships change over time, which will reduce the efficiency of selection. This will not happen with direct selection on QTN.

5. Linkage disequilibrium relationships may be different between populations and thus MAS may not be applicable in populations without GWAS.

6. Allelic frequencies for a marker in LD will not accurately reflect the allelic frequencies of the QTN. As noted previously, this information is very useful, because it gives a horizon for the gain that can be achieved by selection.

7. If the QTN is determined, then selection can also be applied to other populations and breeds, including those populations that have not been analyzed by GWAS (e.g., Kaupe et al., 2004; Goddard et al., 2006; Ron et al., 2006).

Should QTN be treated differently than LD markers in genetic evaluation programs? The problem that only a small fraction of the population will be genotyped will still apply, but it would seem that once a QTN is detected, bias is no longer a factor, and the QTN can be treated as a fixed rather than a random effect (e.g., Baruch and Weller, 2008).

Finally, we should note that investment in breeding programs is unlike other investments, in that the gains are eternal and cumulative. Thus, a relatively small change in the rate of genetic gain can have a huge economic value. As shown in an example given by Weller (1994), for a program with a constant annual investment, net profit will be positive within a 20-yr profit horizon if nominal annual costs are less than 3 -fold the nominal annual gain. Consider the US dairy cattle population with 10,000,000 cows. Current rates of genetic gain are approximately equal to $100 \mathrm{~kg} / \mathrm{yr}$ in terms of economically corrected milk production. Thus, an additional 1\% increase in the rate of genetic gain is approximately equal to $1 \mathrm{~kg} / \mathrm{cow}$ per year. The nominal 
value of this gain is approximately $\$ 0.1$ per cow, or $\$ 1$ million for the entire industry. Thus, an annual investment of $\$ 3$ million in QTN detection can be justified even if it results in only a $1 \%$ increase in the rate of genetic gain.

\section{CONCLUSIONS}

Although genomic selection can increase rates of genetic gain without determination of the causative QTN, determination of these QTN should increase rates of genetic gain and aid in the understanding of the mechanisms through which the trait is affected. Data obtained by GWAS can be used to deduce QTL genotypes of sires via application of the APGD for concordance testing of putative QTN. This together with next-generation sequencing technology will dramatically reduce costs for QTN determination. By complete genome sequencing of 21 sires with many AI sons, it should be possible to determine concordance for all potential QTN detected by GWAS, thus establishing the field of QTNomics.

\section{ACKNOWLEDGMENTS}

This research was supported by grants from the Israel milk marketing board and the European Sixth Research and Technological Development Framework Programme (proposal no. 016250-2 SABRE). Genotyping was performed by A. Schein and N. Avidan (Pharmacogenetics and Translation Medicine Center, The Rappaport Institute for Research in the Medical Sciences, Technion, Haifa, Israel). We acknowledge the support of the SION, Israeli Company for Artificial Insemination \& Breeding Ltd. (Shikmim, Israel), the Israel Cattle Breeders Association (Caesaria Industrial Park, Israel), and the critical reading and input of $\mathrm{M}$. Shani (ARO, The Volcani Center, Bet Dagan, Israel).

\section{REFERENCES}

Aguilar, I., I. Misztal, D. L. Johnson, A. Legarra, S. Tsuruta, and T. J. Lawlor. 2010. Hot topic: A unified approach to utilize phenotypic, full pedigree, and genomic information for genetic evaluation of Holstein final score. J. Dairy Sci. 93:743-752.

Altshuler, D., M. J. Daly, and E. S. Lander. 2008. Genetic mapping in human disease. Science 322:881-888.

Baruch, E., and J. I. Weller. 2008. Incorporation of discrete genotype effects for multiple genes into animal model evaluations when only a small fraction of the population has been genotyped. J. Dairy Sci. 91:4365-4371.

Chamberlain, A. J., H. C. McPartlan, and M. E. Goddard. 2007. The number of loci that affect milk production traits in dairy cattle. Genetics 177:1117-1123.

Cohen-Zinder, M., E. Seroussi, D. M. Larkin, J. J. Loor, A. Everts-van der Wind, J. H. Lee, J. K. Drackley, M. R. Band, A. G. Hernandez, M. Shani, H. A. Lewin, J. I. Weller, and M. Ron. 2005. Identification of a missense mutation in the bovine ABCG2 gene with a major effect on the QTL on chromosome 6 affecting milk yield and composition in Holstein cattle. Genome Res. 15:936-944.

Cole, J. B., P. M. VanRaden, J. R. O'Connell, C. P. Van Tassell, T. S. Sonstegard, R. D. Schnabel, J. F. Taylor, and G. R. Wiggans. 2009. Distribution and location of genetic effects for dairy traits. J. Dairy Sci. 92:2931-2946.

Darvasi, A. 2005. Dissecting complex traits: The geneticists' "Around the world in 80 days". Trends Genet. 21:373-376.

Druet, T. C. S., and A. P. W. de Roos. 2010. Imputation of genotypes from different single nucleotide polymorphism panels in dairy cattle. J. Dairy Sci. 93:5443-5454.

Ducrocq, V., and Z. Liu. 2009. Combining genomic and classical information in national BLUP evaluations. Pages 172-177 in Proc. Interbull Meeting, Barcelona, Spain. Interbull, Uppsala, Sweden.

Georges, M., D. Nielsen, M. Mackinnon, A. Mishra, R. Okimoto, A. T. Pasquino, L. S. Sargent, A. Sorensen, M. R. Steele, X. Zhao, J. E. Womack, and I. Hoeschele. 1995. Mapping quantitative trait loci controlling milk production in dairy cattle by exploiting progeny testing. Genetics 139:907-920.

Goddard, M. E., B. Hayes, H. McPartlan, and A. J. Chamberlain. 2006. Can the same genetic markers be used in multiple breeds? Proc. 8th World Congr. Genet. Appl. Livest. Prod., Belo Horizonte, Brazil. CD-ROM Commun. 22-14.

Goddard, M. E., and B. J. Hayes. 2007. Genomic selection. J. Anim. Breed. Genet. 124:323-330.

Grapes, L., J. C. Dekkers, M. F. Rothschild, and R. L. Fernando. 2004. Comparing linkage disequilibrium-based methods for fine mapping quantitative trait loci. Genetics 166:1561-1570.

Grisart, B., W. Coppieters, F. Farnir, L. Karim, C. Ford, P. Berzi, N. Cambisano, M. Mni, S. Reid, P. Simon, R. Spelman, M. Georges, and R. Snell. 2002. Positional candidate cloning of a QTL in dairy cattle: Identification of a missense mutation in the bovine DGAT1 gene with major effect on milk yield and composition. Genome Res. 12:222-231.

Hayden, E. C. 2009. Genomics shifts focus to rare diseases. Nature 461:458-459.

Hayes, B. J., A. Chamberlain, and M. E. Goddard. 2006. Use of linkage markers in linkage disequilibrium with QTL in breeding programs. Proc. 8th World Cong. Genet. Appl. Livest. Prod., Belo Horizonte (MG), Brazil. CD-ROM Commun. 30-06.

Hickey, J., B. Kinghorn, J. van der Werf, B. Tier, and M. Cleveland. 2010. Recursive long range phasing and long haplotype library imputation: Building a global haplotype library for Holstein cattle. Proc. 9th World Congr. Genet. Appl. Livest. Prod., Leipzig, Germany. CD-ROM Commun. 0934.

Israel, C., and J. I. Weller. 2004. Effect of type I error threshold on marker-assisted selection in dairy cattle. Livest. Prod. Sci. 85:189-199.

Kaupe, B., A. Winter, R. Fries, and G. Erhardt. 2004. DGAT1 polymorphism in Bos indicus and Bos taurus cattle breeds. J. Dairy Res. 71:182-187.

Keane, O. M., N. Maqbool, A. F. McCulloch, J. C. McEwan, and K. G. Dodds. 2009. Generation of a preliminary bovine gene atlas, using expression clustering to annotate gene function. Genet. Mol. Res. 8:1013-1027.

Khatkar, M. S., P. C. Thomson, I. Tammen, and H. W. Raadsma. 2004. Quantitative trait loci mapping in dairy cattle: Review and meta-analysis. Genet. Sel. Evol. 36:163-190.

Lander, E. S., and L. Kruglyak. 1995. Genetic dissection of complex traits: Guidelines for interpreting and reporting linkage results. Nat. Genet. 11:241-247.

Loberg, A., and J. Dürr. 2009. Interbull survey on the use of genomic information. Proc. Interbull Int. Workshop-Genomic Information in Genetic Evaluations. Interbull, Uppsala, Sweden.

Maher, B. 2008. Personal genomes: The case of the missing heritability. Nature 456:18-21.

Mardis, E. R. 2008. The impact of next-generation sequencing technology on genetics. Trends Genet. 24:133-141.

McKay, S., R. Schnabel, B. Murdoch, L. Matukumalli, J. Aerts, W. Coppieters, D. Crews, E. Neto, C. Gill, C. Gao, H. Mannen, P. 
Stothard, Z. Wang, C. Van Tassell, J. Williams, J. Taylor, and S. Moore. 2007. Whole genome linkage disequilibrium maps in cattle. BMC Genet. 8:74.

Meuwissen, T. H. E., and M. E. Goddard. 2000. Fine mapping of quantitative trait loci using linkage disequilibria with closely linked marker loci. Genetics 155:421-430.

Misztal, I., I. Aguilar, A. Legarra, D. Johnson, S. Tsuruta, and T. Lawlor. 2010. A unified approach to utilize phenotypic, full pedigree, and genomic information for genetic evaluation. Proc. 9th World Cong. Genet. Appl. Livest. Prod., Leipzig, Germany. CDRom Commun. 0050.

Ogorevc, J., T. Kunej, A. Razpet, and P. Dovc. 2009. Database of cattle candidate genes and genetic markers for milk production and mastitis. Anim. Gen. 40:832-851.

Olsen, H. G., H. Nilsen, B. Hayes, P. R. Berg, M. Svendsen, S. Lien, and T. Meuwissen. 2007. Genetic support for a quantitative trait nucleotide in the ABCG2 gene affecting milk composition of dairy cattle. BMC Genet. 8:32.

Pérez-Enciso, M., and L. Ferretti. 2010. Massive parallel sequencing in animal genetics: Wherefroms and wheretos. Anim. Genet. 41:561-569.

Ron, M., M. Cohen-Zinder, C. Peter, J. I. Weller, and G. Erhardt. 2006. ABCG2 polymorphism in Bos indicus and Bos taurus cattle breeds. J. Dairy Sci. 89:4921-4923.

Ron, M., and J. I. Weller. 2007. From QTL to QTN identification in livestock-Winning by points rather than knock-out: A review. Anim. Genet. 38:429-439.

Salih, H., and D. L. Adelson. 2009. QTL global meta-analysis: Are trait determining genes clustered? BMC Genomics 10:184.

Sargolzaei, M., F. S. Schenkel, G. B. Jansen, and L. R. Schaeffer. 2008. Extent of linkage disequilibrium in Holstein cattle in North America. J. Dairy Sci. 91:2106-2117.

Szyda, J., A. Żarnecki, and S. Kamiński. 2009. The Polish genomic breeding value estimation project. Proc. Interbull Int. WorkshopGenomic Information in Genetic Evaluations. Interbull, Uppsala, Sweden.

VanRaden, P. M. 2008. Efficient methods to compute genomic predictions. J. Dairy Sci. 91:4414-4423.

VanRaden, P. M., C. P. Van Tassell, G. R. Wiggans, T. S. Sonstegard, R. D. Schnabel, J. F. Taylor, and F. S. Schenkel. 2009. Invited review: Reliability of genomic predictions for North American Holstein bulls. J. Dairy Sci. 92:16-24.

Visscher, P. M. 2008. Sizing up human height variation. Nat. Genet. 40:489-490.

Visscher, P. M. 2010. Genome-wide association studies of complex traits in human populations: Past, present, progress and implications for genomic selection in farm animals. Proc. 9th World Congr. Genet. Appl. Livest. Prod., Leipzig, Germany. CD-Rom Commun. 0973.
Weigel, K. A., G. de los Campos, O. González-Recio, H. Naya, X. L. Wu, N. Long, G. J. M. Rosa, and D. Gianola. 2009. Predictive ability of direct genomic values for lifetime net merit of Holstein sires using selected subsets of single nucleotide polymorphism markers. J. Dairy Sci. 92:5248-5257.

Weigel, K. A., G. de los Campos, A. I. Vazquez, G. J. M. Rosa, D. Gianola, and C. P. Van Tassell. 2010. Accuracy of direct genomic values derived from imputed single nucleotide polymorphism genotypes in Jersey cattle. J. Dairy Sci. 93:5423-5435.

Weller, J. I. 1994. Economic Aspects of Animal Breeding. Chapman \& Hall, London, UK.

Weller, J. I. 2007. Marker assisted selection in dairy cattle. Pages 199-228 in Marker-Assisted Selection, Current Status and Future Perspectives in Crops, Livestock, Forestry and Fish. E. Guimarães, J. Ruane, B. D. Scherf, A. Sonnino, J. D. Dargie, ed. Food and Agriculture Organization of the United Nations, Rome, Italy.

Weller, J. I. 2009. Quantitative Trait Loci Analysis in Animals. 2nd ed. CABI Publishing. London, UK.

Weller, J. I., M. Golik, E. Seroussi, E. Ezra, and M. Ron. 2003. Population-wide analysis of a QTL affecting milk-fat production in the Israeli Holstein population. J. Dairy Sci. 86:2219-2227.

Weller, J. I., Y. Kashi, and M. Soller. 1990. Power of "daughter" and "granddaughter" designs for genetic mapping of quantitative traits in dairy cattle using genetic markers. J. Dairy Sci. 73:25252537.

Weller, J. I., M. Shlezinger, and M. Ron. 2005. Correcting for bias in estimation of quantitative trait loci effects. Genet. Sel. Evol. $37: 501-522$

Weller, J. I., J. Z. Song, D. W. Heyen, H. A. Lewin, and M. Ron. 1998. A new approach to the problem of multiple comparisons in the genetic dissection of complex traits. Genetics 150:1699-1706.

Winter, A., W. Kramer, F. A. O. Werner, S. Kollers, S. Kata, G. Durstewitz, J. Buitkamp, J. E. Womack, G. Thaller, and R. Fries. 2002 Association of a lysine-232/alanine polymorphism in a bovine gene encoding acyl-CoA:diacylglycerol acyltransferase (DGAT1) with variation at a quantitative trait locus for milk fat content. Proc. Natl. Acad. Sci. USA 99:9300-9305.

Yeager, M., Z. Deng, J. Boland, C. Matthews, J. Bacior, V. Lonsberry, A. Hutchinson, L. A. Burdett, L. Qi, K. B. Jacobs, J. GonzalezBosquet, S. I. Berndt, R. B. Hayes, R. N. Hoover, G. Thomas, D. J. Hunter, M. Dean, and S. J. Chanock. 2009. Comprehensive resequence analysis of a $97 \mathrm{~kb}$ region of chromosome $10 \mathrm{q} 11.2 \mathrm{con}-$ taining the MSMB gene associated with prostate cancer. Hum. Genet. 126:743-750.

Zhang, Z., and T. Druet. 2010. Marker imputation with low-density marker panels in Dutch Holstein cattle. J. Dairy Sci. 93:54875494. 